\title{
Effectiveness of Ivermectin in SARS-CoV-2/COVID-19 Patients
}

\author{
Aijaz Zeeshan Khan Chachar , Khurshid Ahmed Khan, \\ Mohsin Asif, Khushbakht, Arous Khaqan, Rabia Basri
}

\begin{abstract}
Background:The first case of Infection with severe acute respiratory syndrome coronavirus 2 (SARSCoV-2) were diagnosed in Wuhan, China in 2019. In the first half of 2020 this disease has already converted into a global pandemic. Objectives: To assess the efficacy of Ivermectin in mild cases of COVID-19 patients on the basis of predefined assessment criteria. Study Settings: Fatima Memorial Hospital, Lahore Study Design: Open label randomized control trial. Duration of Study: From $1^{\text {st }}$ May, 2020 to $30^{\text {th }}$ June, 2020.Patients \& Methods: Sample size and technique: Sample size was 50 patients; 25 patients were kept in control group and 25 patients were kept in experimental group. Results: There were total 50 patients, divided into two groups case and control group.The mean age of the participants was $40.60 \pm 17$ and out of those $31(62 \%)$ were male and $19(38 \%)$ were females. Cough was observed more in case group $(\mathrm{p}=0.049)$. Fever, myalgias and dyspnea were the commonest symptoms in both the groups $(\mathrm{p}=1.000)$. Diarrhea and vomiting were more common in control group $(\mathrm{p}=0.0001, \mathrm{p}=0.042$ respectively). On follow up at day 7, patients were stratified as asymptomatic and symptomatic. Amongst the case group, out of 25 patients, 16(64\%) patients were asymptomatic and rest of the $9(36 \%)$ patients was asymptomatic. In control group, out of 25 patients, $15(60 \%)$ patients were asymptomatic and rest of the $10(40 \%)$ patients was asymptomatic. p-value (0.500). Statistically there was no significant difference between case group who were given ivermectin along with symptomatic treatment and control group who were only given symptomatic treatment without ivermectin, being asymptomatic on day 7 at follow up. p-value (0.500)
\end{abstract}

Keywords: Corona Virus, Covid-19, SARS-COv-2, Antiviral, Ivermectin, Pandemic

\section{Introduction:}

The first case of Infection with severe acute respiratory syndrome coronavirus 2 (SARS-CoV-2) were diagnosed in Wuhan, China in 2019. In the first half of 2020 this disease has already converted into a global pandemic, having different forms of presentation in different patients.

Exponential rise in its cases along with associated mortality has shaken the world. ${ }^{1}$

The severe acute respiratory syndrome coronavirus 2 (SARS-CoV-2) is a single-stranded ribonucleic acid (RNA) virus that causes a severe acute respiratory syndrome. The virus was originally called SARSCoV-2 named officially by World Health Organization as COVID-19 and a global health emergency. The first known case of infection was recorded in early December 2019 and subsequently spread to various continents, including Europe and the United States ${ }^{2,3}$ Generally, 75 percent of patients recover without any notable complication however 25 percent can experience associated complications leading to intensive care unit transfer and even mortality. 4

Ivermectin has different effects to treat variety of diseases and it has proven antimicrobial, antiviral, and anti-cancer properties and considered as a wonder drug. It is highly effective against many microorganisms including some viruses. ${ }^{5}$ Many studies revealed its antiviral effects on RNA viruses such as Zika, dengue, yellow fever, West Nile, Hendra, Newcastle, Venezuelan equine encephalitis, chikungunya, Semliki Forest, Sindbis, Avian influenza A, Porcine Reproductive and Respiratory Syndrome, Human immunodeficiency virus(HIV) type 1, and severe acute respiratory syndrome coronavirus SARS-CoV-2. Fon other hand there are few studies showing antiviral effects of ivermectin against DNA viruses such as Equine herpes type 1, $\mathrm{BK}$ polyomavirus, pseudorabies, porcine circovirus 2 , and bovine herpesvirus $1 .^{5}$ Ivermectin plays a vital role in several biological mechanisms, therefore it could emerge as a potential candidate in the treatment of a wide range of viruses including COVID-19 as well as other types of positive-sense single-stranded RNA viruses. In vivo studies of animal models revealed a broad range of antiviral effects of ivermectin, however, more clinical trials are necessary as it's the need of the hour to do so, as we can validate the potential efficacy of ivermectin in clinical settings.

The recent report by Caly et al. $^{6}$ describing the antiviral potential of ivermectin against the severe

This article is published under the terms of the Creative Commons Attribution License 4.0

Author(s) retain the copyright of this article. Publication rights with Alkhaer Publications.

Published at: http://www.ijsciences.com/pub/issue/2020-09/

DOI: 10.18483/ijSci.2378; Online ISSN: 2305-3925; Print ISSN: 2410-4477 
acute respiratory syndrome coronavirus 2 (SARS$\mathrm{CoV}-2)$ in vitro arrive to the agenda of potential candidates for COVID-19 treatment. ${ }^{6}$

Currently, there is not any Food and drug administration (FDA) approved specific effective antiviral treatment available for COVID-19.Although most of the COVID-19 patients have mild or moderate courses, up to $5 \%-10 \%$ can have severe, potentially life threatening course, there is an urgent need for development of effective \& safe drugs to deal with this novel virus. Optimized supportive care remains the mainstay of therapy. There are more than 300 clinical trials going on, various antiviral and immunomodulating agents are in different stages of evaluation for COVID-19 in those trials and results/ primary end points of few trials will be published in the next couple of months. Despite the urgent need to find an effective and safe antiviral treatment for COVID-19 through randomized controlled studies, certain agents are being used all over the world based on either in-vitro or conclusive evidence or observational studies. $^{7}$

The clinical efficacy and utility of ivermectin in SARS-CoV-2-infected patients is unpredictable at this stage, as we are dealing with a completely novel virus.

There is an urgent need for investigation of the mortality causes and development of novel therapeutic options for severe COVID-19.

\section{Operational definition: COVID-19 infection:}

Patients were labeled to have mild disease due to acute respiratory syndrome coronavirus 2 (SARSCoV-2; previously named as 2019-nCoV) proven via RT-real time COVID-19 polymerase chain reaction (PCR) test.

\section{Methodology: \\ Study Design:}

It was a open label randomized control trial where participants were allocated using predefined protocol who met the inclusion criteria of study after signing a written $\&$ informed consent either by the patient or the person legally authorized to sign.

\section{Study Settings:}

Study was conducted in Fatima Memorial Hospital, Lahore among patients reporting to COVID-19 clinics and medical outpatient department OPD.

\section{Sample size and technique:}

our sample size was 50 patients; 25 patients were kept in control group and 25 patients were in experimental group. Patients were allocated randomly to the groups by computer generated number. Sampling technique was convenient sampling as per the inclusion and exclusion criteria. It was an open label randomized control trial which was done on COVID-19 patients proven by RT- PCR fulfilling the criteria (mild severity).

Duration of study: Starting after IRB approval, $1^{\text {st }}$ May, 2020 to $30^{\text {th }}$ June 2020

\section{Inclusion Criteria:}

- All patients diagnosed with COVID-19 infection with positive reverse transcriptase RT-PCR test, who were willing to participate in this study

- Patients having age of 18-75 years

- Patients of both genders male and female

- Patients who had mild symptoms of Corona virus disease and RT- PCR positive for SARSCov-2

- Ability to take oral medication and were willing to adhere to the drug intake regimen

\section{Exclusion Criteria:}

- Known severe allergic reactions to Ivermectin

- Pregnancy or breastfeeding

- Severe symptoms likely attributed to Cytokine Release Storm

- Malignant diseases

- Chronic kidney disease

- Cirrhosis liver with Child class B or C

Data Collection and analysis Procedure: All patients having COVID-19 proven by RT-PCR reporting to outpatient department OPD and clinics at Fatima memorial Hospital were studied. Patients were allocated randomly to the groups by computer generated number. Data regarding Epidemiological, demographic, clinical, and treatment was collected and analyzed. Outcomes were noted. All the data entered on a structured Performa (Annex-I)

Response of the patients after Ivermectin prescription and response of the patients without Ivermectin prescription was recorded. Response was recorded on the basis of clinical parameters (Fever, Cough, sore throat, Headache, Shortness of breath, lethargy, and fatigue. Any side effects noted after prescription of Ivermectin was recorded.

\section{Ivermectin Prescribing protocol:}

Patients were prescribed Ivermectin $12 \mathrm{mg}$ stat and then $12 \mathrm{mg}$ after 12 hours and $12 \mathrm{mg}$ after 24 hours and we looked at the response at day 7 on follow up in terms of improvement of symptoms like (Fever, Cough, sore throat, Headache, Shortness of breath, lethargy, and fatigue. 
Data was entered and analyzed using SPSS 25.0. Frequency and percentages were calculated for the qualitative variables like gender, comorbidity, symptoms, response to treatment. Quantitative variables of the study like age were expressed as Mean \pm SD

\section{Ethical Considerations:}

Patients were included in the study after written and informed consent. Institutional review board IRB approval was taken from the ethical committee.

\section{Results:}

Total of fifty patients were included in our study, the mean age of the participants was $40.60 \pm 17$ and out of those $31(62 \%)$ were male and $19(38 \%)$ were females. Patients were divided in two groups, case and control. Case group was offered the symptomatic treatment along with Ivermectin and control group was given only symptomatic treatment without Ivermectin. Control group particpants' were older than the case group statistically but there is no difference between the average ages of both groups $(p=0.582)$. There was no association between the gender and case group $(\mathrm{p}=0.561)$. Cough was observed more in case group $(\mathrm{p}=0.049)$. Fever, myalgias and dyspnea were the commonest symptoms in both the groups $(\mathrm{p}=1.000)$. Diarrhea and vomiting were more common in control group ( $\mathrm{p}=0.0001, \mathrm{p}=0.042$ respectively). Regarding loss of taste and and anosmia, were more seen in case group $(\mathrm{p}=0.0009)$

Table: 1 -Age \& Gender distribution of the participants \& Comparative analysis of groups at presentation

\begin{tabular}{lllll}
\hline & Gariables & Group & Total & p-value \\
\cline { 2 - 4 } & Case & Control & $41.84 \pm 15.695$ & 0.582 \\
\hline Age & $40.60 \pm 17$ & $43.08 \pm 14.8$ & $31(62 \%)$ & 0.561 \\
\hline Gender $\quad$ Male & $17(34 \%)$ & $14(28 \%)$ & $19(38 \%)$ & 0.049 \\
\hline Female & $8(16 \%)$ & $11(22 \%)$ & $42(84 \%)$ & 1.000 \\
\hline Cough & $24(48 \%)$ & $18(36 \%)$ & $49(98 \%)$ & 0.128 \\
\hline Fever & $25(50 \%)$ & $24(48 \%)$ & $34(68 \%)$ & 0.072 \\
\hline Sore throat & $20(40 \%)$ & $14(28 \%)$ & $33(66 \%)$ & 0.364 \\
\hline Headache & $20(40 \%)$ & $13(26 \%)$ & $50(100 \%)$ & 0.042 \\
\hline Nauspnea & $25(50 \%)$ & $25(50 \%)$ & $26(32 \%)$ & 0.000 \\
\hline Vomiting & $6(12 \%)$ & $10(20 \%)$ & $20(40 \%)$ & 1.000 \\
\hline Diarrhea & $6(12 \%)$ & $14(28 \%)$ & $47(42 \%)$ & 0.009 \\
\hline Myalgia & $4(8 \%)$ & $17(34 \%)$ & $20(40 \%)$ & 0.009 \\
\hline Loss of taste & $23(46 \%)$ & $24(48 \%)$ & $20(40 \%)$ & 0.247 \\
\hline Anosmia & $15(30 \%)$ & $5(10 \%)$ & $8(16 \%)$ & \\
\hline Hypotension & $15(30 \%)$ & $5(10 \%)$ & $6(12 \%)$ & \\
\hline
\end{tabular}

Age is presented as Mean \pm SDand for comparison independent sample t test is applied

All the categorical variables are presented in N (\%) and Fisher's Exact Test is applied

On follow up at day 7, patients were stratified as asymptomatic and symptomatic. Amongst the case group, out of 25 patients, $16(64 \%)$ patients were asymptomatic and rest of the $9(36 \%)$ patients was asymptomatic. In control group, out of 25 patients, $15(60 \%)$ patients were asymptomatic and rest of the $10(40 \%)$ patients was asymptomatic. p-value $(0.500)$ statistically there is no significant difference between case group who were given ivermectin along with symptomatic treatment and control group who were only given symptomatic treatment without ivermectin, being asymptomatic on day 7 at follow up. p-value (0.500) Table-2

In case group, adverse reaction of ivermectin like heartburn was seen in $8(32 \%)$ patients and reported as well.
Table-2: Comparative analysis of groups on Follow up at day 7

\begin{tabular}{|c|c|c|c|}
\hline Group & \multicolumn{2}{|c|}{ follow up @Day 7} & \\
\hline & Asymptomatic & Symptomatic & Total \\
\hline Case & $16(64 \%)$ & $9(36 \%)$ & 25 \\
\hline Control & $15(60 \%)$ & $10(40 \%)$ & 25 \\
\hline Total & $31(62 \%)$ & $19(38 \%)$ & $50(100 \%)$ \\
\hline
\end{tabular}

Discussing about the comorbidities, out of all these 50 patients, there were $20(40 \%)$ patients who had diabetes mellitus, $11(22 \%)$ in case group and $9(18 \%)$ in control group. Hypertension was seen in $13(26 \%)$ patients, $7(14 \%)$ in case group and $6(12 \%)$ in control group. There were $6(12 \%)$ obese patients, $2(\% 4)$ in case group and in $4(8 \%)$ in control group. Cardiovascular disease was observed in $4(8 \%)$ patients and each group had $2(4 \%)$ patients. There were $15(30 \%)$ patients who were active smokers, 9 $(18 \%)$ in case group and $6(12 \%)$ in control group. There was no patient with chronic lung disease in our study (Table-3) 
Table: 3.Frequency of comorbidities

\begin{tabular}{|c|c|c|c|}
\hline Comorbidity & Total & Case group & Control group \\
\hline $\mathrm{DM}^{*}$ & $20(40 \%)$ & $11(22 \%)$ & $9(18 \%)$ \\
\hline $\mathrm{HTN}^{+}$ & $13(26 \%)$ & $7(14 \%)$ & $6(12 \%)$ \\
\hline Obesity & $6(12 \%)$ & $2(4 \%)$ & $4(8 \%)$ \\
\hline CVD $^{\$}$ & $4(8 \%)$ & $2(4 \%)$ & $2(4 \%)$ \\
\hline Smoking & $15(30 \%)$ & $9(18 \%)$ & $6(12 \%)$ \\
\hline
\end{tabular}

$\mathrm{DM}^{*}$ Diabetes mellitus, $\mathrm{HTN}^{+}$Hypertension, $\mathrm{CVD}^{\$}$

Cardiovascular disease

\section{Discussion:}

In this study, Effectiveness of Iveremectin was assessed among the participants by looking at the symptoms on day 7 at follow up. To best of our knowledge, very few studies of this kind had been conducted in our region. We report that patients on presentation had fever, myalgias being the commonest symptoms found in both groups' patients presenting to our hospital.

Regarding recovery at Day 7 on follow up, case group vs control group, $16(64 \%)$ Vs 15 (60\%) and a study conducted in Bangladesh ${ }^{8}$ revealed recovery in terms of being symptom free was seen in 55\% patients which is comparable with our study results, however the study conducted in Bangladesh was a comparative study between two groups receiving two different treatment regimens, one group received ivermectin with doxycycline and other group received azithromycin with hydroxychloroquine.

A study conducted by Patel $A^{9}$ on 169 critically sick patients in early days of Covid-19 showed survival benefit in $18.3 \%$ of patients, however this study took all the critical patients of Covid-19 and in contrast to our study which was only conducted on mild cases of COVID-19 and our study revealed that after giving Ivermectin, on day 7,64\% patients were symptom free (recovery). A huge difference in recovery between our study and the study conducted by Patel $\mathrm{A}^{9}$ can be attributed to the patients' selection in both the studies. We selected only mild disease patients with Covid-19 whereas study by Patel $\mathrm{A}^{9}$ selected all the critically sick patients including mechanically ventilated patients.

A huge multicentre study named as ICON (Ivermectin in Coronavirus disease nineteen) conducted in Covid-19 patients in South Florida ${ }^{10,11}$ enrolled 280 admitted patients and mean age of the participants was $59.6 \pm 17.9$ years and our study participants mean age was $42 \pm 17$ years which is comparable to some extent as we only took out patients and study by Rajter $\mathrm{JC}^{10}$ only enrolled all the admitted patients, so elder patients got admitted more.

In the study conducted by Rajter $\mathrm{JC}^{10}$ there were two groups, one treated with ivermectin and other group was given conventional treatment other than
Ivermectin, there was statistically significant difference between two groups' mortality $15 \%$ vs $25 \%$ and also mortality was also lower in 75 patients having severe pulmonary disease treated who were given ivermectin $(38.8 \%$ vs $80.7 \%)$. Ivermectin group showed less mortality during treatment of COVID-19, especially in patients who were on higher inspired oxygen or ventilatory support. In contrast our study was first of its kind in our country conducted on outpatients having mild disease, showed recovery in $64 \%$ patients.

COVID-19 pandemic has affected over 200 countries in a span of less than 4 months burdening on the entire health care systems of many countries with no FDA approved drug which can stop the viral transmission or infectivity. Most of the drugs which are being used to treat this novel virus are not so effective and the development of vaccines is under preparation and may be available on trial basis in future, therefore, there is urgent need of the day to develop and use drugs cautiously. Ivermectin which is famous and used frequently as antiparasitic drug but in recent past studies have shown that Ivermectin has antiviral activity. It is easily available in our country and highly affordable as well. Ivermectin is officially approved for use in SARS-cov patients in few countries like Peru and Bolivia. ${ }^{12}$

COVID-19 has hit the whole world resulting in a significant mortality as well as leaving a huge impact on every country's economy. Various treatment options are still under study. Recently ivermectin's activity against SARS-CoV-2 gives ray of hope, but off-label and compassionate use requires careful risk-benefit considerations, especially in critically ill patients. A path to consider is evaluation first of impacts on virologic outcomes in uncomplicated, low-risk patients early in the course of the disease. Well-conducted clinical trials informed by robust pharmacokinetic models should be considered to validate the impact before the use of ivermectin to treat SARS-CoV-2 is implemented.

\section{Conclusion:}

Our study didn't show statistically any significant difference between case and control group. In Ivermectin's (case group) recovery was almost equal to control group who received only conventional symptomatic treatment, so this is the need of the day that we need to conduct more randomized controlled trials across our country involving major tertiary care health care facilities with larger sample size to assess its efficacy for validating the use of Ivermectin against SARS-CoV-2. Nearly 40 clinical trials are ongoing world over for measuring the outcome of COVID-19 treatment with Ivermectin. 


\section{References:}

1. World Health Organization. Coronavirus disease 2019 (COVID-19): situation report, 72.

2. Sohrabi C, Alsafi Z, O'Neill N, Khan M, Kerwan A, Al-Jabir A, Iosifidis C, Agha R. World Health Organization declares global emergency: A review of the 2019 novel coronavirus (COVID-19). International Journal of Surgery. $2020 \mathrm{Feb}$ 26.3.https://doi.org/10.1016/j.ijsu.2020.03.036

3. Lu H, Stratton CW, Tang YW. Outbreak of pneumonia of unknown etiology in Wuhan, China: The mystery and the miracle. Journal of medical virology. 2020 Apr;92(4):401-25. https://doi.org/10.1002/jmv.25678

4. Bai Y, Yao L, Wei T, Tian F, Jin DY, Chen L, Wang M. Presumed asymptomatic carrier transmission of COVID-19. Jama. $\quad 2020 \quad$ Apr $\quad 14 ; 323(14): 1406-7$. https://doi.org/10.1001/jama.2020.2565

5. Heidary F, Gharebaghi R. Ivermectin: a systematic review from antiviral effects to COVID-19 complementary regimen. The Journal of Antibiotics. 2020 Jun 12:1-0. https://doi.org/10.1038/s41429-020-0336-z

6. Caly L, Druce JD, Catton MG, Jans DA, Wagstaff KM. The FDA-approved drug ivermectin inhibits the replication of SARS-CoV-2 in vitro. Antiviral research. 2020 Apr 3:104787. https://doi.org/10.1016/j.antiviral.2020.104787

7. YAVUZ S, Ünal S. Antiviral treatment of COVID-19. Turkish Journal of Medical Sciences. 2020 Apr 21;50(SI1):611-9. https://doi.org/10.3906/sag-2004-145
8. 8.Chowdhury AT, Shahbaz M, Karim MR, Islam J, Dan G, Shuixiang H. A comparative observational study on Ivermectin-Doxycycline and HydroxychloroquineAzithromycin therapy on COVID19 patients. https://doi.org/10.21203/rs.3.rs-38896/v1

9. Patel A. Usefulness of Ivermectin in COVID-19 Illness. Available at SSRN 3580524. 2020 Apr 19. https://doi.org/10.1101/2020.06.06.20124461

10. Rajter JC, Sherman M, Fatteh N, Vogel F, Sacks J, Rajter JJ ICON (Ivermectin in COvid Nineteen) study: Use of Ivermectin is Associated with Lower Mortality in Hospitalized Patients with COVID19. medRxiv. 2020 Jan 1. https://doi.org/10.26434/chemrxiv.11875446.v1

11. Cennimo David J. What is the role of antiparasitic drug ivermectin in the treatment of corona virus disease 2019 (Covid-19)? https://www.medscape.com/answers/2500114197513/what-is-the-role-of-the-antiparasitic-drug-ivermectinin-the-treatment-of-coronavirus-disease-2019-covid19 Updated: Jul 02, https://doi.org/10.26434/chemrxiv.11875446.v1

12. Ivermectin Usage Accelerates While the Need for Data is Real: How About an Ivermectin Registry? MAY 26, 2020 BANGLADESH, BOLIVIA, COVID-19, IVERMECTIN, PERU, POPULAR POSTS, SARS-COV-2. 